\title{
ORTHOGONAL ISOMORPHIC REPRESENTATIONS OF FREE GROUPS
}

\section{J. DE GROOT}

1. Introduction. We consider the group \& of proper orthogonal transformations (rotations) in three-dimensional Euclidean space, represented by real orthogonal matrices $\left(a_{i k}\right)(i, k=1,2,3)$ with determinant +1 . It is known that this rotation group $\$(5)$ contains free (non-abelian) subgroups; in fact Hausdorff (5) showed how to find two rotations $P$ and $Q$ generating a group with only two non-trivial relations

$$
P^{2}=Q^{3}=I \text {. }
$$

Now the elements $P Q P Q$ and $P Q^{2} P Q^{2}$ are free generators of a free rotation group $R(7)$. It was shown in (4), starting from $R$ and using transfinite induction, that (S) contains even a free subgroup with continuously many free generators. $^{1}$

Now it is clear that this method for constructing free subgroups of $(5)$ is an indirect one and furnishes only special free rotation groups. These disadvantages became more visible in certain problems (partly geometrical, partly group-theoretical) dealing with free rotation groups in spaces of dimension $>3$. Therefore we shall develop in this paper a straightforward and simple method of determining free subgroups of (S) (we restrict ourselves, however, to the three-dimensional case). The only, but in many cases serious, difficulty with this type of problem is to prove time and again that certain products of matrices do not vanish identically. As our main result (Theorem II) we shall give, explicitly, continuously many rotations (with the same rotation angle, and rotation axes situated in the same plane), which are free generators of a free group (of continuous rank). Other representations of free groups were given by Fuchs-Rabinowitsch (3) and Doniakhi (2; see also Sanov 8), who use two-rowed square matrices; however, these cannot be orthogonal. Some conjectures are stated in $\$ 5$.

\footnotetext{
Received March 23, 1955.

${ }^{1}$ After completion of this paper, the author heard from Poland that Sierpinski proved a lemma $(9,238)$ which, though not stated in terms of group theory, implies the existence of a free rotation group of countable rank, and from which the existence of a free rotation group of continuous rank can easily be deduced. Already, Sierpiński uses in his proof the "von Neumann numbers" (see $\$ 4$ of this paper). On the other hand we see that Sierpiński's proof essentially makes use of the Hausdorff result (5), just as does the proof in (4). For this reason the methods derived in the present paper improve those in (9) and (4) and Theorems I and II cannot be obtained by employing the methods of (9), (4) only.
} 
2. Preliminaries. We consider polynomials $V$ in the variables $\sin n \phi$, $\cos m \phi$ ( $n, m$ ranging over the integers) over the real field. Each term

$$
\prod_{i} \sin ^{\tau_{i}} n_{i} \phi \cdot \prod_{j} \cos ^{s_{i}} m_{j} \phi
$$

has degree

$$
\sum_{i}\left|r_{i} n_{i}\right|+\sum_{j}\left|s_{j} m_{j}\right| .
$$

The degree of $V$ is the maximum of the degrees of each of its terms.

Lemma I. A polynomial $V$, having only one term of degree equal to the degree of $V$ itself, is a non-constant function of $\phi$.

Proof. Using the formulae

$$
\begin{aligned}
\sin n \phi & =\left(\begin{array}{l}
n \\
1
\end{array}\right) \cos ^{n-1} \phi \sin \phi-\left(\begin{array}{l}
n \\
3
\end{array}\right) \cos ^{n-3} \phi \sin ^{3} \phi+\ldots \\
& =\left[\left(\begin{array}{l}
n \\
1
\end{array}\right)+\left(\begin{array}{l}
n \\
3
\end{array}\right)+\ldots\right] \cos ^{n-1} \phi \sin \phi+\ldots \\
\cos m \phi & =\cos ^{m} \phi-\left(\begin{array}{c}
m \\
2
\end{array}\right) \cos ^{m-2} \phi \sin ^{2} \phi+\ldots \\
& =\left[1+\left(\begin{array}{c}
m \\
2
\end{array}\right)+\left(\begin{array}{c}
m \\
4
\end{array}\right)+\ldots\right] \cos ^{m} \phi+\ldots
\end{aligned}
$$

$V$ is transformed into a polynomial in $\sin \phi$ and $\cos \phi$, again having only one term of maximal degree. This expression of $V$ can obviously be written in the form

$$
\sum_{i=0}^{k}\left(\alpha_{i} \sin \phi+\beta_{i} \cos \phi\right) \cos ^{k-i} \phi+\gamma
$$

either $\alpha_{0}$ or $\beta_{0}$ being equal to zero.

If $k=0$ this polynomial does not vanish identically in $\phi$. Then the lemma follows by induction. Suppose the lemma holds for $k-1$; suppose that for the value $k$ the polynomial $V \equiv 0$. Substituting $\phi= \pm \frac{1}{2} \pi$ in (1) we find $\alpha_{k}=\gamma=$ 0 ; thus we can divide $V$ by $\cos \phi$, and get a contradiction. So $V \not \neq 0$, and $V$ is non-constant.

LEMмa II. Consider the real orthogonal matrices

$$
A(\phi)=\left(\begin{array}{ccc}
\cos \phi & -\sin \phi & 0 \\
\sin \phi & \cos \phi & 0 \\
0 & 0 & 1
\end{array}\right), B(\phi)=\left(\begin{array}{ccc}
1 & 0 & 0 \\
0 & \cos \phi & -\sin \phi \\
0 & \sin \phi & \cos \phi
\end{array}\right) .
$$

Any proper ${ }^{2}$ product in terms of $A$ and $B$ is a non-constant matrix (depending on $\phi)$.

${ }^{2}$ The product is proper if it cannot be transformed into the unity-matrix $I$ using only the trivial relations $A \cdot A^{-1}=B \cdot B^{-1}=I, A \cdot I=I \cdot A=A, B \cdot I=I \cdot B=B$. 
Proof. At first we prove the Lemma for products of the form

$$
A^{n_{1}} B^{m_{1}} A^{n_{2}} B^{m_{2}} \ldots A^{n_{k}} B^{m_{k}} \quad\left(n_{i}, m_{i} \text { integers } \neq 0\right)
$$

If $k=1$, we get

$$
A^{n_{1}} B^{m_{1}}=\left(\begin{array}{ccc}
\cos n_{1} \phi & -\sin n_{1} \phi \cos m_{1} \phi & \sin n_{1} \phi \sin m_{1} \phi \\
\sin n_{1} \phi & \cos n_{1} \phi \cos m_{1} \phi & -\cos n_{1} \phi \sin m_{1} \phi \\
0 & \sin m_{1} \phi & \cos m_{1} \phi
\end{array}\right) .
$$

This is obviously a non-constant matrix. (2) is a matrix $\left(a_{i j}\right)$. Denote the degree of $a_{i j}$ by $d_{i j}$. Suppose these degrees satisfy for $k=l-1$ the relations

$$
\left\{\begin{array}{l}
d_{11}, d_{21} \leqslant \sum_{i=1}^{k}\left|n_{i}\right|+\sum_{i=1}^{k-1}\left|m_{i}\right|, \\
d_{12}, d_{13}, d_{22}, d_{23}=\sum_{i=1}^{k}\left(\left|n_{i}\right|+\left|m_{i}\right|\right),
\end{array}\right.
$$

while, moreover, each of the elements $a_{12}, a_{13}, a_{22}, a_{23}$ has exactly one term of the corresponding degree denoted in (3).

Now one sees easily by multiplying $\left(a_{i j}\right)$ (for $k=l-1$ ) with

$$
A^{n_{b}} B^{m_{k}}
$$

that $\left(a_{i j}\right)$ (for $k=l$ ) satisfies the same conditions. Since this is also true for $k=1$, the Lemma follows by induction, applying Lemma I, for all products of type (2).

If we multiply (2) on the right by

$$
A^{n_{k+1}}
$$

we see also in the same way-using the properties mentioned-that this product depends on $\phi$. Since the interchanging of $A$ and $B$ in (2) does no harm, the Lemma is proved.

Remark. In the proof it is possible, but not necessary, to consider the whole of the matrix $\left(d_{i k}\right)$; one could also deal with the degrees of the second row only. One might also consider the degree of the trace of $\left(a_{i k}\right)$ (independence of the chosen coordinate-system).

3. Countable representations. Using the well-known substitution

$$
\phi=2 \arctan x
$$$$
(0<\phi<\pi) \text {, }
$$

which yields

$$
\sin \phi=\frac{2 x}{1+x^{2}}, \quad \cos \phi=\frac{1-x^{2}}{1+x^{2}},
$$

the expression (2) is rationalized in terms of $x$. If $x$ is transcendental, we call $\phi$ associated transcendental. 
Now we can state

THEOREM I. The rotation group generated by the rotations $A(\phi)$ and $B(\phi)$, these two being rotations with rotation angle $\phi$ and with rotation axes perpendicular to each other, is a free (non-abelian) group (of rank two) for any fixed, associated transcendental value $\phi$.

Proof. It follows from Lemma II that any element (2) of the group $H$, generated by $A=A(\phi)$ and $B=B(\phi)$, is a non-constant matrix, if it cannot be transformed into identity by using the trivial relations. Now any nontrivial relation in $H$ can be written in the form

$$
A^{n_{1}} B^{m_{1}} A^{n_{2}} B^{m_{\mathbf{s}}} \ldots A^{n_{k}} B^{m_{k}}=I,
$$

the product being a proper product if $k>0$. Since (2) is a non-constant function of $\phi,(6)$ can be transformed, using (4) in a finite number of algebraic equations in $x$, not all vanishing identically. So substituting for $\phi$ any fixed associated transcendental number $\phi$, no relation (6) is valid, and the theorem is proved.

Since in a free group generated by $A$ and $B$ the elements $A^{i} B A^{-i}(i=0,1$, $2, \ldots)$ are free generators of a free group of infinite (but countable) rank, we get

COROLlary. For any fixed associated transcendental $\phi$ the rotations with rotation angle $\phi$ and with rotation axes in the same plane and making angles $i \phi(i=0,1,2, \ldots)$ with a fixed line in this plane are free generators of a free group (of infinite rank).

4. Uncountable representations. J. von Neumann (6) proved that the set $\left\{x_{t}\right\}=M$ of distinct real numbers $x_{t}$, defined by

$$
x_{t}=\sum_{n=0}^{\infty} 2^{2^{[n t]}-2^{n^{*}}}
$$

are algebraically independent over the field of rational numbers (no finite set $\left\{a_{i}\right\}$ of distinct numbers $a_{i} \in M$ satisfies an equation $P\left(y_{i}\right)=0$, if $P\left(y_{i}\right)$ is a non-vanishing polynomial in the variables $y_{i}$ with rational coefficients). Thus there are continuously many, distinct, associated transcendental numbers

$$
\phi_{t}=2 \arctan x_{t} \quad(0<t<1) .
$$

Select another $\phi=\psi$ defined by $\psi=\phi_{t}$ with $t \geqslant 1, t$ fixed.

Now we shall prove

THEOREM II. The continuously many rotations $R_{t}=A\left(\phi_{t}\right) B(\psi) A^{-1}\left(\phi_{t}\right)$ $(0<t<1)$ are free generators of a free rotation group of continuous rank. 
We note that all $R_{t}$ are rotations with the same rotation angle $\psi$ and rotation axes in the same plane. ${ }^{3}$ In particular, the existence of a free rotation group of continuous rank has been established (without using the axiom of choice).

Proof. The theorem is proved if any proper product $P\left(R_{t}\right)$ of a finite number of rotations $R_{t}$ is unequal to the unity matrix. After simplifications we may write

$$
\begin{array}{r}
P\left(R_{t}\right)=A\left(\phi_{t_{1}}\right) B^{k_{2}}(\psi) A^{-1}\left(\phi_{t_{1}}\right) A\left(\phi_{t_{2}}\right) B^{k_{2}}(\psi) A^{-1}\left(\phi_{t_{2}}\right) \ldots \\
A\left(\phi_{t_{n}}\right) B^{k_{n}}(\psi) A^{-1}\left(\phi_{t_{n}}\right),
\end{array}
$$

the $k_{i}$ being integers $\neq 0$, the $t_{i}(i=1,2, \ldots, n)$ real numbers with

$$
0<t_{i}<1, \quad t_{i} \neq t_{i+1} \quad(i=1,2, \ldots, n-1) .
$$

Now replace in the right-hand side of (8) $\psi$ by a real variable $\phi$, and

$$
\phi_{t_{i}}
$$

by a multiplicity $m_{i} \phi$ of $\phi\left(m_{i}\right.$ integers $\left.>0\right)$ with $m_{i}=m_{j}$ if and only if $t_{i}=t_{j}$. After carrying out the simplifications

$$
A^{-1}\left(m_{i} \phi\right) A\left(m_{i+1} \phi\right)=A\left(l_{i} \phi\right)=A^{l_{i}}(\phi) \quad\left(l_{i} \neq 0\right),
$$

(8) is transformed into a proper product (almost) of type (2), thereforeapplying Lemma 2 -into a non-constant matrix function of $\phi$. From this it follows that at least one of the elements $a_{i k}$ of matrix (8) is a non-constant function of

$$
\phi_{t_{i}} \text { and } \psi,
$$

if we consider these for a moment as real variables. But then it is impossible -using substitution (7) and the result of von Neumann - that this function is equal to 0 or 1 if we substitute for the variables (9) their permitted associated transcendental and distinct values. So $P\left(R_{t}\right) \neq I$.

4.1. It is not necessary, of course, to take the rotation axes in the same plane to get free generators. The proof, just established, furnishes us a general method of generating free groups in the following way. Any rotation $R$ can be written as a product

$$
R=\left(\begin{array}{ccc}
\cos \phi_{u} & -\sin \phi_{u} & 0 \\
\sin \phi_{u} & \cos \phi_{u} & 0 \\
0 & 0 & 1
\end{array}\right)\left(\begin{array}{ccc}
1 & 0 & 0 \\
0 & \cos \phi_{v} & -\sin \phi_{v} \\
0 & \sin \phi_{v} & \cos \phi_{v}
\end{array}\right)\left(\begin{array}{ccc}
\cos \phi_{w} & -\sin \phi_{w} & 0 \\
\sin \phi_{w} & \cos \phi_{w} & 0 \\
0 & 0 & 1
\end{array}\right)
$$

${ }^{3}$ One might ask whether the geometrical structure of this set of rotation axes in the plane can be relatively simple, if we select a suitable set of continuously many values $t$. Indeed, it is possible that this set of rotation axes corresponding to the generating rotations is perfect. This follows easily from the fact that the set of numbers $\left\{x_{t}\right\}$ contains perfect subsets. To prove this, we observe that $x_{t}$ is a monotonically increasing function of $t$; thus the set of transcendental numbers $\left\{x_{t}\right\}$ is nowhere dense in the set of all real numbers, and is, moreover, a $G_{\delta}$-set; therefore it contains perfect subsets. 
by electing suitable $\phi_{u}, \phi_{v}, \phi_{w}$ (Eulerian angles, see (1, p. 104)). Let the $u, v, w$ range as real variables over certain sets, say $0<u<1,1<v<2,2<w<3$.

Now we consider elements $R$ corresponding with triplets $(u, v, w)$ differing from each other in each of the variables $u, v$, w. Then the elements are free generators of a free group. Indeed, a version of Lemma II on any proper product can be applied after simplifications.

Briefly the proper products do not vanish identically as functions, and cannot therefore be equal to unity for permitted values of their variables, since these values are algebraically independent.

4.2. Remarks. If we consider $A(\phi)$ and $B(\phi)$ as matrix functions (the elements being analytic functions of the real variable $\phi$ ) it follows from Lemma II and Theorem I that these matrix functions are free generators of a free group (the only constant function in the group being the unity matrix).

In a certain analogy with the generators of Theorem II, one can also consider the family of matrix functions

$$
A\left(\phi_{\alpha}\right) B(\psi) A^{-1}\left(\phi_{\alpha}\right),
$$

the indices $\alpha$ ranging over a set of arbitrary potency $m$ ( $\psi$ and $\phi_{\alpha}$ being real variables). Therefore these orthogonal matrix functions (9) are free generators of a free group of rank $m$. Any free group can therefore be represented isomorphically by a system of orthogonal matrix functions. Perhaps this may be of some use for the theory of free groups.

5. Conjectures. One could try to prove Theorem I by the alternative method of expanding $\sin \phi$ and $\cos \phi$ in a Taylor series.

Writing

$$
\sin x=x+o\left(x^{2}\right), \quad \cos x=1-\frac{1}{2} x^{2}+o\left(x^{2}\right),
$$

one sees easily that for small $x$ all products of type (2) with $k \leqslant 2$ give a non-constant matrix. But this fails already in the case $k=3$; taking, for example

$$
n_{1}=2, n_{2}=3, n_{3}=-5, \quad m_{1}=-5, m_{2}=2, m_{3}=3 .
$$

However, for $k \leqslant 3$, a proof is possible if we expand $\sin x$ and $\cos x$ up to $o\left(x^{3}\right)$. It will perhaps be possible to get a proof of Theorem I using induction; however, the computations involved are very lengthy. On the other hand, it may be possible to generalize this method in cases where the generating rotations $A$ and $B$ are not perpendicular to each other. Consider

$$
A^{\prime}=C A C^{-1} \text { with } C=B(\alpha)
$$

for a fixed but arbitrary $\alpha$ and carry out the computations for products (2), in which $B$ is replaced by $A^{\prime}$. This gives the following conjecture (generalizing Theorem I): two rotations with arbitrary but different rotation axes are free 
generators of a free group for all rotation angles $\phi$, a countable number of values $\phi$ excepted.

We conclude with another conjecture: Let $\left\{\mathfrak{S}_{\alpha}\right\}$ be a family of at most continuously many groups $\mathcal{S}_{\alpha}$, each of which is countable (or more generally consists of less than continuously many elements) and can be represented as a three-dimensional rotation group; now the free product of the groups $\mathbb{S}_{\alpha}$ can be isomorphically represented by a rotation group.

\section{REFERENCES}

1. L. Bieberbach, Analytische Geometrie (Teubner, 1932).

2. Kh. A. Doniakhi, Linear representation of the free product of cyclic groups, Leningrad State Univ. Annals [Uchenye Zapiski] Math. Ser., 10 (1940), 158-165 (Russian).

3. D. J. Fuchs-Rabinowitsch, Leningrad State Univ. Annals [Uchenye Zapiski] Math. Ser., 10 (1940), 154-157 (Russian).

4. J. de Groot and T. Dekker, Free subgroups of the orthogonal group, Comp. Math., 12 (1954), 134-136.

5. F. Hausdorff, Grundzüge der Mengenlehre (1914), 469-472.

6. J. von Neumann, Ein System algebraisch unabhängiger Zahlen, Math. Ann., 99 (1928), 134-141.

7. R. M. Robinson, On the decomposition of spheres, Fund. Math., 34 (1947), 246-260.

8. I. N. Sanov, $A$ property of a representation of a free group, Doklady Akad. Nauk. SSSR (N.S.), 57 (1947), 657-659 (Russian).

9. W. Sierpiński, Sur le paradoxe de la sphère, Fund. Math., 93 (1945), 235-244.

\section{Univer sity of Amsterdam}

